Résumés des conférences et travaux

\title{
Histoire des sciences dans l’Occident médiéval
}

\author{
Nicolas Weill-Parot
}

\section{OpenEdition \\ Journals}

Édition électronique

URL : https://journals.openedition.org/ashp/3067

DOI : $10.4000 /$ ashp.3067

ISSN : 1969-6310

Éditeur

Publications de l'École Pratique des Hautes Études

\section{Édition imprimée}

Date de publication : 1 septembre 2019

Pagination : 268-270

ISSN : 0766-0677

Référence électronique

Nicolas Weill-Parot, "Histoire des sciences dans l'Occident médiéval », Annuaire de l'École pratique des hautes études (EPHE), Section des sciences historiques et philologiques [En ligne], 150 | 2019, mis en ligne le 11 juin 2019, consulté le 06 juillet 2021. URL : http://journals.openedition.org/ashp/3067 DOI : https://doi.org/10.4000/ashp.3067 


\title{
HISTOIRE DES SCIENCES DANS L'OCCIDENT MÉDIÉVAL
}

\author{
Directeur d'études : M. Nicolas WeILL-PAROT
}

Programme de l'année 2017-2018 : I. Le vol dans les airs au Moyen Âge : étude d'une utopie scientifique. - II. Commentaires de la Physique d'Aristote des universités d'Europe centrale (milieu du XIVe siècle-milieu du XVe siècle).

\section{Le vol dans les airs au Moyen Âge : étude d'une utopie scientifique}

L'un des thèmes du séminaire pour l'année 2016-2017 portait sur l'utopie scientifique au Moyen Âge. Cette année c'est l'une de ces « utopies scientifiques » qui a été retenue pour le premier thème : le rêve de vol humain. Les préhistoires ou les épistémologies du vol humain (Jules Duhem, Clive Hart, Wolfgang Behringer et Constance Ott-Koptschalijski, Daniel Parrochia...) accordent le plus souvent une place relativement limitée à la période médiévale qui, de fait, compte peu de tentatives de ce genre. Nous avons souhaité mener une histoire conceptuelle du vol humain médiéval compris comme « utopie scientifique ». L'enquête comprenait deux versants : d'une part, l'analyse des conceptions scientifiques du vol humain que ses principes soient scientifico-techniques, magiques ou miraculeux, d'autre part, la recherche dans le cadre scientifique lui-même des paramètres susceptibles de venir à l'appui des possibilités d'un vol, en particulier la question des corps lourds se maintenant ou se déplaçant dans l'air.

Nous avons commencé par analyser les occurrences du topos consistant à illustrer l'impossible par l'exemple de l'homme qui vole chez l'astrologue Albumasar dans son Liber introductorii maioris ad scientiam iudiciorium astrorum, traduit en latin au $\mathrm{XII}^{\mathrm{e}}$ siècle, et chez Thomas d'Aquin, qui rappelle ce motif pour donner un exemple d'une évidence de nature, pour montrer que ce n'est pas une déficience, pour indiquer que le libre arbitre ne peut rien y changer, ou pour étudier la proposition conditionnelle : "si l'homme vole, alors il a des ailes ».

Nous avons analysé les rares témoignages de conceptions effectives ou rêvées d'instruments pour voler. Nous sommes partis de la machine imaginée par Roger Bacon dans plusieurs de ses écrits. Dans l'Epistola de secretis operibus artis et naturae, il signale que cette invention extraordinaire, à la différence des autres qu'il énumère, n'a pas encore été réalisée. Il exclut donc implicitement le légendaire transport aérien d'Alexandre le Grand installé dans une nacelle traînée par des griffons : il veut, en effet, promouvoir une machine autonome s'affranchissant de toute force animale et démultipliant la force humaine par un mécanisme.

L'autre grand exemple se situe avant l'ère de la science scolastique : la tentative de vol du moine anglais du XI ${ }^{\mathrm{e}}$ siècle Eilmer de Malmesbury. Nous avons repris l'analyse du passage où le chroniqueur Guillaume de Malmesbury rapporte l'événement, étudié jadis par Lynn White, en proposant de le situer dans le récit. Des parallèles nous sont apparus avec le passage de la comète de Halley signalé juste avant l'insertion de ce récit. La question des sources d'Eilmer et de Guillaume a été examinée. Le modèle du vol d'Icare et de Dédale invoqué par le chroniqueur a donné lieu aussi à 
une enquête. Enfin, le récit du vol d'Ibn Firnas (IX ${ }^{\mathrm{e}}$ siècle), auquel on a souvent comparé celui d'Eilmer, a été évoqué à la lumière de travaux récents.

La connaissance des mécanismes du vol des oiseaux est une question majeure, mais peu détaillée dans les sources médiévales : nous avons repris l'analyse des textes commentant ou complétant ce qu'en dit Aristote dans ses œuvres zoologiques, notamment le traité de fauconnerie de Frédéric II et le De animalibus d'Albert le Grand.

Le vol du magicien (Simon le Magicien, trône de Salomon) ou, plus tard, celui des sorciers (thème renouvelé par les travaux de Martine Ostorero), est perçu comme un vol porté par les démons, ce qui a donné lieu à des réflexions aux confins de la théologie et de la philosophie naturelle dignes d'intérêt. Le long développement sur la capacité des démons à transporter des corps déployé dans le De malo de Thomas d'Aquin a été analysé. Nous nous sommes aussi tournés vers les récits hagiographiques présentant des vols portés par les anges comme cela est attesté, par exemple, dans la Vie de sainte Hildegonde rapportée par Césaire d'Heisterbach. En revanche, le motif de l'ange volant grâce à ses ailes, récurrent dans l'Écriture elle-même, dans la littérature et dans l'iconographie, se heurte aux implications philosophiques de leur incorporéité. Dans la philosophie scolastique les rapports au lieu de l'ange, conçu comme une intelligence séparée, suscite des spéculations complexes mises en lumière par les travaux de Tiziana Suarez Nani et de son équipe, rendant inapplicable l'idée de vol. Les éléments d'explication de la lévitation de saints comme Thomas d'Aquin et Marguerite de Hongrie, que l'on trouve dans les sources hagiographiques, évoquent aussi l'âme transportant elle-même le corps.

La seconde partie de l'étude était consacrée aux textes de philosophie naturelle. Le magnétisme (avec le morceau de fer susceptible, malgré sa gravité, de s'élever vers l'aimant) et l'horreur du vide (avec l'expérience de la chantepleure, où l'eau ne tombe pas alors qu'elle est plus lourde que l'air) offraient des exemples de corps lourds se maintenant dans l'air. À partir d'un passage du De anima d'Aristote, Walter Burley dans son Commentaire de la Physique, en discutant, à propos du problème de l'horreur du vide, l'expérience des deux surfaces planes en contact qu'on ne peut écarter, déduit qu'entre deux corps solides il y a toujours de l'air et donc admet la proposition, qui n'est qu'en apparence une « bouffonnerie », selon laquelle les animaux marchent toujours sur de l'air ou de l'eau.

La comparaison entre vol et natation étant souvent attestée, la question de la natation et de la flottaison d'un corps a été analysée, ce qui a conduit à un rappel de l'histoire de la connaissance du principe de la poussée d'Archimède, jadis retracée par Marshall Clagett. C'est effectivement soit en ignorant ce principe soit en en tenant compte qu'Albert de Saxe et Nicole Oresme imaginent une sorte d'aérostat rempli de feu qui naviguerait au-dessus de la sphère de l'air, expérience de pensée bien connue des historiens de la préhistoire du vol humain. L'expérience de l'outre gonflée d'air chaud évoquée par Albert le Grand (et mise en exergue jadis par Jules Duhem) à propos d'un passage difficile du Traité $d u$ ciel d'Aristote (IV.4, 311b1-14) nous a conduits à suivre diverses interprétations (Pierre d'Auvergne, Jean Buridan, Albert de Saxe, Nicole Oresme). Enfin, nous nous sommes intéressés à l'interprétation du mouvement du projectile, pour comprendre si la substitution au XIV ${ }^{\mathrm{e}}$ siècle de l'explication par l'impetus à l'explication aristotélicienne par l'air mû allait ou non dans le sens de l'idée de vol artificiel. 
Durant cette année, Marion Lieutaud, docteur de l'université Paris IV-Sorbonne, a exposé l'interprétation par Giordano Bruno de la lévitation de saint Thomas d'Aquin.

Ce thème du vol humain au Moyen Âge, complété par des recherches ultérieures, doit donner lieu à la publication prochaine d'un livre.

\section{Commentaires de la Physique d'Aristote des universités d'Europe centrale (milieu du XIV siècle-milieu du XVé siècle)}

L'année 2016-2017 avait été consacrée à l'étude des relations entre physique et logique dans le commentaire inédit du maître oxonien d'origine germanique Johannes Scharpe. Cette année, l'enquête s'est tournée vers le corpus des commentaires de la Physique dans les nouvelles universités d'Europe centrale. L'historiographie a souvent soutenu trop vite que l'intérêt de ces textes était faible, leur contenu dégradé se contentant de suivre sans grande originalité l'école de Jean Buridan, d'Albert de Saxe et de Marsile d'Inghen. Mes précédentes recherches dans les fonds de la bibliotheca amploniana conservée à l'université Erfurt m'avaient permis de nuancer la sévérité de ce jugement. Parmi les commentaires de la Physique conservés, l'un m'avait semblé particulièrement digne d'intérêt, celui d'un maître presque inconnu Roger (Rudigerus) Dole de Roermundia ( $†$ 1409). Formé à l'université de Paris, comme beaucoup de ses confrères, il se transfère à la faculté des arts de Vienne et y enseigne de 1385 à 1400, étudiant la théologie à partir de 1396. Son Exercitium librorum Physicorum, daté de 1395, est conservé dans le ms. Ampl. Q. 302. Le texte est peu lisible et des passages sont parfois effacés par l'humidité. Roger Dole est manifestement influencé, comme les autres maîtres de sa génération, par les commentaires de Jean Buridan, d'Albert de Saxe et de Marsile d'Inghen. Nous nous sommes donc d'abord penchés sur ces contributions modèles pour la question «le moteur et le mû sont-ils toujours ensemble » posée régulièrement dans les commentaires du début du livre VII et qui donne lieu à l'examen de toute une série de cas qui semblent défier cet axiome « contiguïste » aristotélicien. L'approche de la question par le maître est relativement singulière. Nous avons ensuite étudié le passage consacré au vide (livre IV) où il reformule l'explication de l'évitement du vide à partir de la nécessité pour l'influence céleste d'emprunter la ligne la plus droite. La «nature commune » (ou universelle) intervient ordinairement pour rendre compte de phénomènes qui contredisent la « nature particulière » comme l'horreur du vide ou la génération des monstres. Nous avons suivi (livre II) la recherche de définition de cette nature commune, ainsi que l'explication de la génération des monstres et les rôles respectifs de la nature particulière et de la nature universelle que nous avons comparés avec ceux qu'indiquent les trois commentaires modèles.

Roger Dole n'apparaît pas comme un simple compilateur des commentaires antérieurs. Et si son commentaire, à visée d'enseignement, ne présente pas d'innovations comparables à celles d'un Jean Buridan, il semble maîtriser en profondeur les enjeux des questions, connaître fort bien leur tradition, et être capable de nouvelles formulations qui en font un grand maître. Il témoigne donc de l'existence d'un enseignement d'un niveau soutenu dans l'une de ces universités germaniques nouvellement fondées, offrant par là même un indice de la qualité de la science «normale ». 